\title{
Analysis of chaotic saddles in a nonlinear vibro-impact system
}

\author{
Jinqian Feng $^{\dagger}$ \\ School of Science of Xi'an Polytechnic University, Xi'an 710048, People's Republic China
}

\begin{abstract}
In this paper, a computational investigation of chaotic saddles in a nonlinear vibro-impact system is presented. For a classical Duffing vibro-impact oscillator, we employ the bisection procedure and an improved stagger-and-step method to present evidence of visual chaotic saddles on the fractal basin boundary and in the internal basin, respectively. The results show that the period saddles play an important role in the evolution of chaotic saddle. The dynamics mechanics of three types of bifurcation such as saddle-node bifurcation, chaotic saddle crisis bifurcation and interior chaotic crisis bifurcation are discussed. The results reveal that the period saddle created at saddle-node bifurcation is responsible for the switch of the internal chaotic saddle to the boundary chaotic saddle. At chaotic saddle crisis bifurcation, a large chaotic saddle can divide into two different chaotic saddle connected by a period saddle. The intersection points between stable and unstable manifolds of this period saddle supply access for chaotic orbits from one chaotic saddle to another and eventually induce the coupling of these two chaotic saddle. Interior chaotic crisis bifurcation is associated with the intersection of stable and unstable manifolds of the period saddle connecting two chaotic invariant sets. In addition, the gaps in chaotic saddle is responsible for the fractal structure.
\end{abstract}

Keywords: Duffing vibro-impact oscillator; Chaotic saddle; Period saddle; Crisis bifurcation

\section{Introduction}

Non-smooth dynamical systems (NDSs) are frequently encountered in applied science and engineering, including mechanical systems with impacts [1-2], piecewise-smooth systems with switches in electronic circuits [3], and hybrid control systems [4]. That is, their motions are characterized by smooth evolutions interrupted by instantaneous non-smooth events such as impacting, switching, sliding and other

\footnotetext{
${ }^{\dagger}$ Corresponding author (J.Q. Feng).

E-mail address: jqfeng15@126.com \& fengjinqian@mail.nwpu.edu.cn.
} 
transitions. There are mature literatures that draw attentions to NDSs, including the work of Kunze [5], Leine and Nijmeijer [6], di Bernardo et al. [7] and Luo [8]. NDSs often exhibit complicated and novel dynamics, e.g. periodic-adding (PD) cascades [9] and so called non-smooth bifurcations [10-11].

Previous literatures are concerned with the local analysis of persistent and attracting solutions. Recently, Mason et al. computed the basin of attraction and grazing-induced bifurcation in a non-smooth models [12-13]. However, chaotic transients occurred in NDSs has aroused less interest. In practice, chaotic transients exist extensively in the impact oscillator. These systems all strongly suggest the existence of chaotic saddles. As is well known, chaotic saddles known as non-attracting chaotic sets are notably responsible for the fractal basin boundary, chaotic transients and scattering [14-15].

This paper is organized as follows. In Sec. 2, the interesting model with a unilateral rigid barrier is described as a typical Duffing vibro-impact system. In Sec. 3, an improved strategy is proposed to approach computationally the chaotic saddles. In Sec. 4 , based on the analysis of chaotic saddles, we investigate the dynamics of saddle-node ( $\mathrm{SN}$ ) bifurcation, chaotic saddle crisis (CSC) bifurcation and interior chaotic crisis (ICC) bifurcation. Conclusions are given in Sec. 5.

\section{The model and equations of motion}

Assume that the mass-spring model shown in Fig. 1 is forced by the harmonic excitation. The equation of motion of the mass with a unilateral rigid barrier is of the form

$$
m \ddot{z}+k_{1} z+k_{2} z^{3}+c \dot{z}=\mu \cos (\Omega \tau), \quad z<\Delta,
$$

where the overdots denote the derivative with respect to $\tau . c$ is the linear damping coefficient, $\mu$ and $\Omega$ represent the external forcing amplitude and frequency, respectively. In the rigid barrier $z=\Delta$, there is an instantaneous impact according to Newton's law, the classical instantaneous impact law is 


$$
\dot{z}_{+}=-R \dot{z}_{-}, \quad z=\Delta
$$

the parameter $R$ is the restitution factor which contributes to impact losses, $\dot{z}_{+}$and $\dot{z}_{-}$refer to the velocity just before and after the impact, respectively.

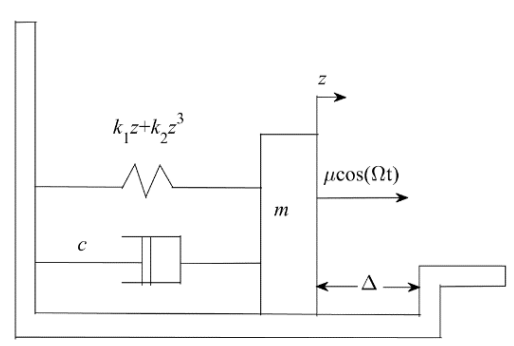

Fig. 1. The vibro-impact model subjected to harmonic excitation.

The corresponding dimensionless equations of motion can be rewritten with a scale time $t=\varpi \tau$ in Eqs. (1) and (2) as

$$
\left\{\begin{array}{l}
\ddot{x}+\alpha_{1} x+\alpha_{2} x^{3}+\beta \dot{x}=f \cos (\omega t), H(x)<0, \\
\dot{x}_{+}=-R \dot{x}_{-}, H(x)=0 .
\end{array}\right.
$$

where $\varpi^{2}=m, \alpha_{1}=k_{1}, \alpha_{2}=k_{2}, \quad \beta=\frac{c}{\varpi^{2}}, f=\mu, \omega=\frac{\Omega}{\varpi}, x=z$, and the impacting boundary function is given by

$$
H(x)=x-\Delta .
$$

Obviously, the hybrid system (3) denotes a typical Duffing vibro-impact system.

Let $X=(x, \dot{x})^{T}$, the period stroboscopic Poincare map can be given as

$$
F: X(t) \rightarrow X(t+T)
$$

where $T=\frac{2 \pi}{\omega}$ is the external driver period. Using the map (5), the system (3) can be rewritten in the discrete map form as

$$
X_{n+1}=F\left(X_{n}\right) .
$$

In the following study, numerical simulations of the system (6) are performed by adopting the integral schemes in Ref. [16]. 


\section{Approaching chaotic saddles}

Both chaotic attractor and chaotic saddle are strange invariant sets. Usually these two invariant sets are described as the union of points where stable and unstable manifold of saddle-point solutions cross each other. However, chaotic attractor is closed but chaotic saddle is open. It is a difficult task to obtain numerically the chaotic saddle because of its inherent quality of non-attracting, that is, any numerical trajectory starting near the stable manifold, no matter how close, will be first attracted to the chaotic saddle and eventually be repelled along its unstable manifold. However, note that the closer the initial condition is to its stable manifold, the longer time it stays in the chaotic saddle. A variety of numerical algorithms have been developed to compute the so-called pseudo saddle trajectory near the chaotic saddle, such as the sprinkler method [17], the PIM method [18], the PIM-simplex method [19] and the standard stagger-and-step method (SM) [20]. In this section, an improved SM will be proposed to compute the arbitrarily long trajectory in a small scale $\varepsilon$ neighborhood of the chaotic saddle.

\subsection{The standard SM}

For the discrete map (6), let $D \subset R^{n}$ be the interested region which is divided into many uniform rectangular small grids named as cells. Each cell has a unique cell number associated with it. By introducing a suitable restraining region $S \subset D$ which contains a chaotic saddles and no attractor, the escape time can be defined as the minimum number $n \geq 0$ for which the $n$th iteration is not in $S$, that is,

$$
T(X)=\min \left\{n \geq 0: F^{n}(X) \notin S\right\} .
$$

Let $T(X)=\infty$ if $F^{n}(X) \in S$ for all $n \geq 0$. Thus the set of phase points $X$ such that $T(X)=\infty$ constitutes an invariant set that contains the chaotic saddle. For a given escape time threshold $T^{*}>>1$ and any initial condition $X_{0} \in S$ with $T\left(X_{0}\right) \geq T^{*}$, the Ref. [20] suggests the following stagger-and-step iteration 


$$
X_{n+1}=\left\{\begin{array}{cl}
F\left(X_{n}\right), & T\left(X_{n}\right)>T^{*}, \\
F\left(X_{n}+r_{n}\right), & T\left(X_{n}\right) \leq T^{*},
\end{array}\right.
$$

where $r_{n}$ is a randomly selected stagger and satisfies $\left\|r_{n}\right\|<\delta$. The direction of $r_{n}$ is chosen randomly with uniform distribution on a $N$ sphere. Once the condition $T\left(X_{n}\right) \leq T^{*}$ in iterative formula (8) is reached at the $n$th iteration, the random search interposes and stops until the stagger $r_{n}$ satisfies $T\left(X_{n}+r_{n}\right)>T^{*}$. The phase point $X_{n}$ is then renewed as $X_{n}=X_{n}+r_{n}$. Using the algorithm, the iterative formula (8) can generate a $\delta$-pseudo-trajectory $\left\{X_{n}: \mathrm{n} \geq 0\right\}$ such that $\left\|F\left(X_{n}\right)-X_{n+1}\right\|<\delta$ with $T\left(X_{n}\right)>\mathrm{T}^{*}$ for all $n \geq 0$. This trajectory $X_{n}$ can stay in the region $S$ for arbitrarily long times. Thus it can approach eventually the saddle along its stable manifold after a few transient iterations.

As mentioned in Ref. [20], a proper threshold $T^{*}$ plays a key role in implementing successfully SM. Note that the larger threshold $T^{*}$ leads to the longer time during which the trajectory $X_{n}$ lies within the chaotic saddle. Thus, a sufficiently large threshold $T^{*}$ is necessary in order to make sure that the trajectory $X_{n}$ is closer adequately to its stable manifold of the chaotic saddle. However, the successful probability $\left\{P\left(X_{n}\right): T\left(X_{n}\right)>T^{*}\right\}$ decays exponentially as the threshold $T^{*}$ increases, which implies that the larger threshold $T^{*}$ increase the difficulty for searching successfully $X_{n}$ with $T\left(X_{0}\right)>T^{*}$. Extraordinarily, no staggers $r_{n}$ may exist if the threshold $T^{*}$ is too large.

In order to give a good choice for the threshold $T^{*}$, the average escape time $\langle\tau\rangle$ is introduced as follows:

$$
\langle\tau\rangle=\lim _{t \rightarrow \infty} \lim _{N_{0} \rightarrow \infty} \frac{\ln \left(N_{0} / N_{t}\right)}{t},
$$

where $N_{t}$ represents the number of trajectories that are still in the region $S$ after $t$ 
iterations, and $N_{0}$ is the number of initial point with a uniform distribution in $S$. The Ref. [20] suggests that the admissible threshold $T^{*}$ must be large compare to $\langle\tau\rangle$ as long as it is in a reasonable range.

\subsection{The improved SM}

The saddle-type pseudo-trajectory may also be produced by the standard SM if the chosen threshold $T^{*}$ based on $\langle\tau\rangle$ is not large enough. Assume that for a certain successful stagger $r_{n}, T\left(X_{n}+r_{n}\right)$ is much greater than $T^{*}$, that is, the difference $\Delta T=T\left(X_{n}+r_{n}\right)-T^{*}$ is large enough. According to the iterative formula (8), the continuous iterations with no staggers may proceed for $\Delta T$ steps. If the trajectory stays on the stable manifold, the numerical procedure is not sensitive to the choice of $T^{*}$. Those continuous iterations with $\Delta T$ steps can still make sure that the trajectory lies on the stable manifold. However, the trajectory once meets the edge of chaotic saddle filled unstable solutions, in the succeeding $\Delta T$ steps, may escape from the chaotic saddle along the unstable directions of the unstable solution, eventually, approaches its stable manifold due to the staggers and return again the chaotic saddle.

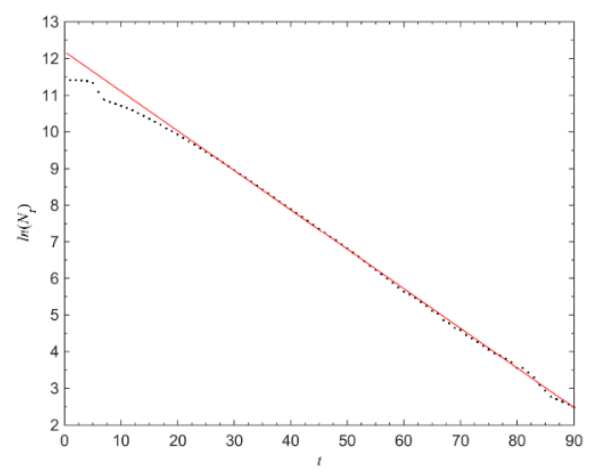

Fig. 2. Scaling of the average escape time $\langle\tau\rangle$.

As an illustration, the system (3) is studied for the fixed parameters

$$
\alpha_{1}=-0.5, \quad \alpha_{2}=1, \quad \beta=0.3, f=0.5, \quad R=0.8, \Delta=1 \text {. }
$$


The frequency is chosen as $\omega=1.14$. Let $(x, y)=(x, \dot{x})$ in Eq.(3), the interested region $D=\{(x, y):-2 \leq x \leq \Delta,-2 \leq y \leq 2\}$ is divided uniformly into $200 \times 200$ cells. The remaining region $S$ can be obtained by excluding the cells that contain attractors. The number of initial points is $N_{0}=300 \times 300$ in formula (9), Fig. 2 shows the variety of $\ln N_{t}$ versus $t$. The slope of the fitted line (red line in Fig.2) is $\gamma \approx-1.05 \times 10^{-1}$. Then the average escape time is $\langle\tau\rangle=-\frac{1}{\gamma} \approx 10$.

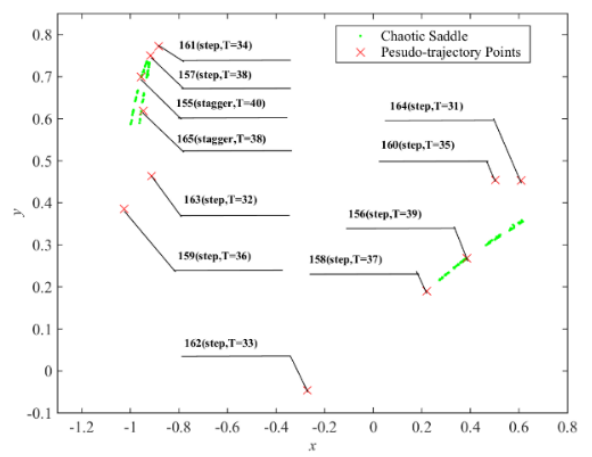

Fig.3. A chaotic saddle with small size is created by the standard SM with a fixed threshold $T^{*}=30$. The green dots depict the chaotic saddle. The red crosses are pseudo-trajectory which embed in the chaotic saddle. The red points from 157 th to 164 th are false trajectory.

Choose the parameters $T^{*}=30>\langle\tau\rangle$ and $\delta=10^{-10}$ in the standard SM, a small chaotic saddle with two branches is shown in Fig. 3. The green dots depict the chaotic saddle. The red crosses are labeled the order of iterations, the type of iteration and the escape time. Those crosses supply insight into the evolution of trajectory escaping from the edge of chaotic saddle. It can be seen from Fig. 3 that, the escape time at the 155 th iteration reaches $T=40>T^{*}$ by a successful stagger. Then the continuous iterations with steps follow until the escape time drops down to $T=T^{*}$ at the 165th iteration, they consist of pseudo-trajectory. Obviously, the iterations from 157 th to 164 th break away from the chaotic saddle, those points are false trajectory. Eventually, the trajectory returns again to the chaotic saddle with the help of 
successful stagger at the $165 t h$ iteration. A new escape time $T=38$ is then updated at the 165 th iteration. Note that, it is important to choose a suitable number of steps after a successful stagger.

Based on the average escape time $\langle\tau\rangle$, an algorithm of self-correcting threshold $T^{*}$ is proposed in the improved SM. Firstly, assume that an initial point $X_{0}$ with $T\left(X_{0}\right) \geq T_{0}^{*}$ is selected. For the $n$th stagger, a maximal step number are defined as

$$
\Delta T_{n}=\min \left\{\Delta T_{n, 1}, \Delta T_{n, 2}\right\}
$$

where $\Delta T_{n, 1}=T_{n}^{s}-T_{n}^{*}$, and $T_{n}^{*}$ depict the referenced threshold at the $n$th iteration. $T_{n}^{s}$ represents the escape time detected successfully at the $n$th iteration, that implies $T_{n}^{s}>T_{n}^{*} . \Delta T_{n, 2}$ is a designated regulator at the $n$th iteration. An updated threshold $T_{n+1}^{*}$ at the next iteration can be adjusted as

$$
T_{n+1}^{*}=T_{n}^{s}-\Delta T_{n}
$$

Obviously, an unabated threshold sequence $\left\{T_{n}^{*}: n=0,1,2, \cdots\right\}$ is then yielded by the iterative formula (12). Not that, $\Delta T_{n}$ dominates the maximal permissible number of step following a successful stagger, that is, $\Delta T_{n}$ represents the number of continuous step between two adjacent staggers. The procedure is illustrated in Fig. 4.

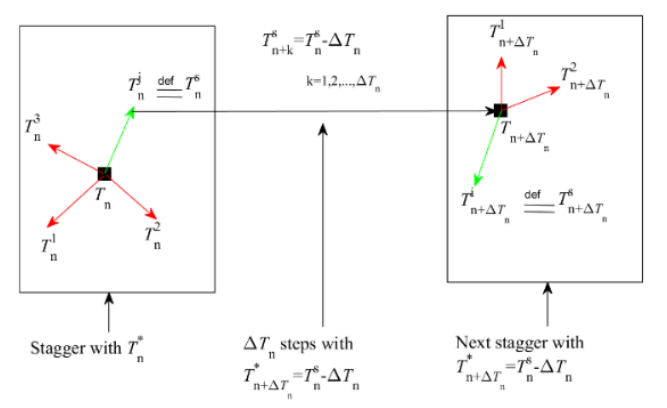

Fig.4. Algorithm of the self-correcting threshold.

In Fig. $4, T_{n}=T\left(X_{n}\right) . T_{n}^{k}(k=1,2, \ldots)$ represents the escape time of the $k$ th tentative stagger at the $n$th random search. The failed and successful staggers are 
identified by the red and green arrows, respectively. When $T_{n} \leq T_{n}^{*}$, a series of staggers will interpose and stop until a successful stagger satisfies $T_{n}^{j}>T_{n}^{*}$ at the $j$ th search. The corresponding escape time $T_{n}^{j}$ of this successful stagger is appointed as $T_{n}^{S}$. Then $\Delta T_{n}$ steps follow with the different thresholds $T_{n+k}^{*}\left(k=1,2, \cdots, \Delta T_{n}\right)$, see formula (12). Once the escape time $T_{n+\Delta T_{n}}$ drops down to $T_{n+\Delta T_{n}}^{*}$ at the $(n+$ $\left.\Delta T_{n}\right)$ th iteration. Next stagger associated with a new threshold $T_{n+\Delta T_{n}}^{*}$ is then carried out.

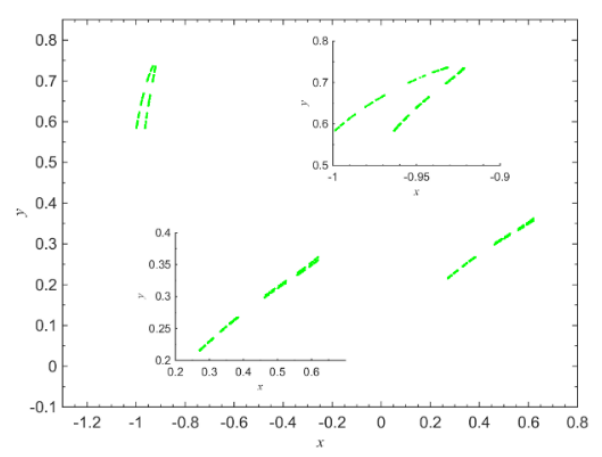

Fig. 5. A robust chaotic saddle is plotted by the improved SM with local enlargements.

In the improved SM the sequence $\left\{T_{n}^{*}: n=0,1,2, \cdots\right\}$ is increased constantly at each stagger, while $T^{*}$ is fixed in the standard SM. However, it is not likely to increase arbitrarily large in the actual procedure. It is reasonable to introduce a critical level $T_{c}$. Once a certain $T_{n}^{*}>T_{c}$ holds, we fix $T_{k}^{*}=T_{c}$ for all $k>n$. As is illustrated of the proposed algorithm, the same parameters are chosen as Fig. 3. But the initial threshold is $T_{0}^{*}=20$, which is smaller than $T^{*}=30$ in Fig. 3. The critical level is $T_{c}=100$. Comparing with Fig. 3, Fig. 5 shows the same chaotic saddle using the improved SM. The graph is created with 1000 points after discarding the first 100 points. It can be seen from Fig. 3 and 5, the chaotic saddle in Fig. 5 is more robust and contains the longer saddle trajectory. Moreover, the algorithm of self-correcting threshold prevents the temporary escape from chaotic saddle, and the false trajectory points vanish. 


\section{Analysis of chaotic saddles}

In this section, the bisection procedure and the improved SM are employed to investigate chaotic saddles on the basin boundary and inside the basins of some attractors, respectively. The external excitation frequency $\omega$ of system (3) is considered, and other system parameters are given in (10). Fig. 6 shows the global bifurcation diagram when the frequencies $\omega$ varies from 1.13 to 1.15 . For each frequency $\omega, 100$ initial points are chosen randomly in the interested region $D$. For each initial point, 300 period points are recorded after discarding the first 500 period points. Those period points indicate the attractors. On the other hand, the period saddles are created by the shooting method. The attractors and saddles are marked as the blue and red dots, respectively. The symbol $A_{j}^{(i)}\left(S_{j}^{(i)}\right)$ represents the $j$ th attractor (or saddle) with period $i$, respectively. When $i=\infty, \mathrm{A}_{j}^{(\infty)}\left(\mathrm{S}_{j}^{(\infty)}\right)$ denotes the chaotic attractor (or chaotic saddle).

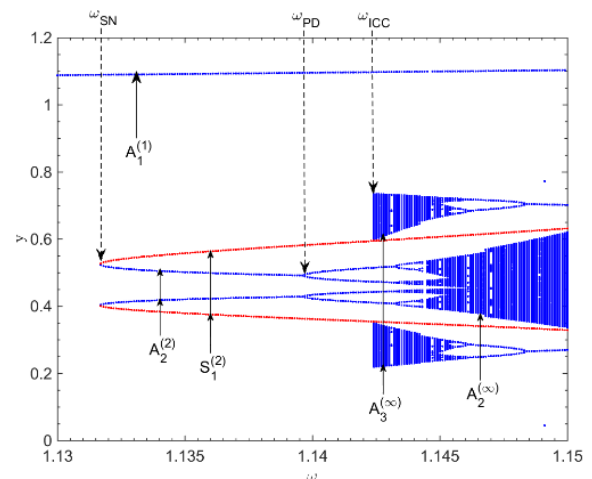

Fig. 6. Global bifurcation diagram with multi-initial points.

In Fig. 6 , when $1.13<\omega<\omega_{\mathrm{SN}} \approx 1.1317$, system (3) possesses a unique attractor $A_{1}^{(1)}$. Its topological characteristic always remain unchanged during $\omega \in[1.13,1.15]$. As the frequency $\omega$ increases to $\omega_{\mathrm{SN}}$, a new period attractor $A_{2}^{(2)}$ and a period saddle $\mathrm{S}_{1}^{(2)}$ emerge simultaneously. The corresponding Floquet multipliers of saddle $S_{1}^{(2)}$ are $(0.02,0)$ and $(1.10,0)$, while attractor $A_{2}^{(2)}$ associates 
its Floquet multipliers with $(0.03,0)$ and $(0.90,0)$. The Floquet theory implies that $\mathrm{SN}$ bifurcation occurs for $\omega_{\mathrm{SN}} \approx 1.1317$. Subsequently, as the continuous increase of the frequency $\omega$, attractor $A_{2}^{(2)}$ undergoes a PD bifurcation cascade starting $\omega=$ $\omega_{\mathrm{PD}} \approx 1.1395$ and eventually leading to a chaotic attractor (denoted by $\mathrm{A}_{2}^{\infty}$ ). For $\omega=\omega_{\text {ICC }} \approx 1.1424$, another chaotic attractor $A_{3}^{\infty}$ appears suddenly. It disappears gradually through an inverse PD bifurcation cascade. When $\omega=\omega_{\text {ICC }}$, chaotic attractor $A_{3}^{\infty}$ consisting of two narrow branches is close to saddle $S_{1}^{(2)}$. This is a typical chaotic crisis bifurcation. Note that, the chaotic saddle is responsible for the appearance of chaotic attractor. The following remarks are interested in the analysis of bifurcations associated with chaotic saddles.

\subsection{SN bifurcation}

In this subsection, SN bifurcation will be investigated in detail. In Fig. 7, cell
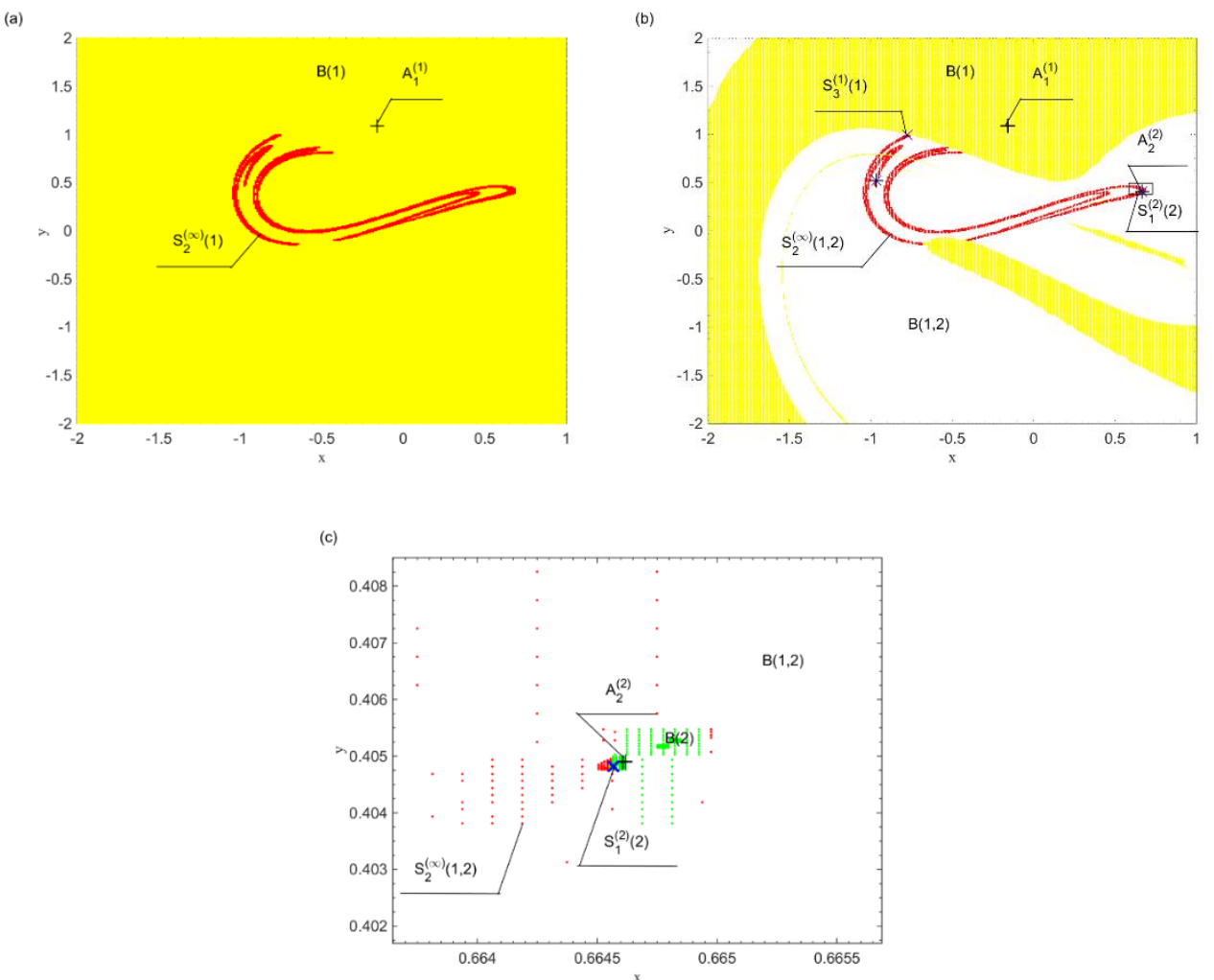

Fig. 7. Global properties for (a) $\omega=1.1316<\omega_{\mathrm{SN}}$, (b) $\omega=1.1317 \approx \omega_{\mathrm{SN}}$. (c) is the local enlargement of the rectangle area of (b). 
mapping associated with the improved SM are applied for global analysis of dynamical system (3). The chosen region is defined by $D=\{(x, y):-2 \leq x \leq \Delta,-2 \leq y \leq 2\}$ with partition $300 \times 400$. The symbol $\mathrm{B}(i)$ represents the basin of attraction of attractor $\mathrm{A}_{i}$, while $\mathrm{B}\left(i_{1}, i_{2}, \cdots, i_{k}\right)$ means the join basin boundary of multiple attractor $A_{i_{1}}, A_{i_{2}}, \cdots, A_{i_{k}} . \mathrm{S}_{j}^{(i)}\left(i_{1}, i_{2}, \cdots, i_{k}\right)$ represents the $j$ th saddle with period $i$ loaded on the basin boundary $\mathrm{B}\left(i_{1}, i_{2}, \cdots, i_{k}\right)$. The attractors and period saddles are marked by black "+" and blue " $X$ " respectively.

For $\omega$ is close to $\omega_{\mathrm{SN}}$, Fig. 7 shows the global properties of system (3). For $\omega=1.1316<\omega_{\mathrm{SN}}$, there are a period attractor $A_{1}^{(1)}$ and a coexisting interior chaotic saddle (ICS) $S_{2}^{(\infty)}(1)$ (red dots), see Fig. 7(a). For $\omega=1.1317 \approx \omega_{\text {SN }}$ in Fig 7(b), right after the $\mathrm{SN}$ bifurcation, attractor $\mathrm{A}_{2}^{(2)}$ and saddle $\mathrm{S}_{1}^{(2)}(2)$ appear synchronously. ICS $S_{2}^{(\infty)}(1)$ keeps its original shape but switches to a boundary chaotic saddle (BCS) $S_{2}^{(\infty)}(1,2)$ (red dots). In order to identify attractor $A_{2}^{(2)}$ and saddle $S_{1}^{(2)}(2)$, the iterative cell mapping method is applied to show the refined dynamical structure. The corresponding local enlargement are given in Fig. 7(c). In addition, the boundary characteristics of $\operatorname{BCS} S_{2}^{(\infty)}(1,2)$ cause the slight fractal structure and induce the birth of a new saddle $S_{3}^{(1)}(1)$, see Fig. 7(b).

When $\omega=1.1317 \approx \omega_{\mathrm{SN}}$, Fig. $7(\mathrm{~b})$ shows clearly that saddle $S_{1}^{(2)}(2)$ and $\mathrm{S}_{3}^{(1)}(1)$ are close to the boundary of $\operatorname{BCS} S_{2}^{(\infty)}(1,2)$. In order to investigate their dynamical relation, stable and unstable manifolds of saddles $S_{3}^{(1)}(1)$ and $S_{1}^{(2)}(2)$ are exhibited in Fig. 8(a) and (b), respectively. The green dots and red dots represent stable manifold $W_{k}^{S}$ and unstable manifold $W_{k}^{u}$ of saddles $k\left(k=\mathrm{S}_{3}^{(1)}(1), \mathrm{S}_{1}^{(2)}(2)\right)$. It is clear that from Fig. 8(a) and (b), both outlines are highly similar between one branch of unstable manifold $\left(W_{S_{3}^{(1)}(1)}^{u}\right.$ in Fig. 8(a) and $W_{S_{1}^{(2)}(2)}^{u}$ in Fig. 8(b)) and BCS 
$S_{2}^{(\infty)}(1,2)$ in Fig. 7(b). Moreover, one branch of stable manifold $W_{S_{3}^{(1)}(1)}^{S}\left(W_{S_{1}^{(2)}(2)}^{S}\right)$ and unstable manifold $W_{S_{3}^{(1)}(1)}^{u}\left(W_{S_{1}^{(2)}(2)}^{u}\right)$ intersects transversally each other. This intersection implies the occurrence of infinite twine, which can induces BCS $\mathrm{S}_{2}^{(\infty)}(1,2)$. On the other hand, another branches of unstable manifolds of saddles $S_{3}^{(1)}(1)$ and $S_{1}^{(2)}(2)$ link to attractor $A_{1}^{(1)}$ and $A_{2}^{(2)}$, respectively, see Fig $8(a)$ and (c). This two unstable manifolds practically supply the two different escape directions for $\operatorname{BCS} S_{2}^{(\infty)}(1,2)$, which eventually causes that $\operatorname{BCS} S_{2}^{(\infty)}(1,2)$ is attracted to two different attractors $\left(A_{1}^{(1)}\right.$ and $\left.A_{2}^{(2)}\right)$ and switches to a boundary status. Reasonably, the saddle created by SN bifurcation is notably responsible for the switch between the ICS (in Fig. 7(a)) and BCS (in Fig. 7(b)).
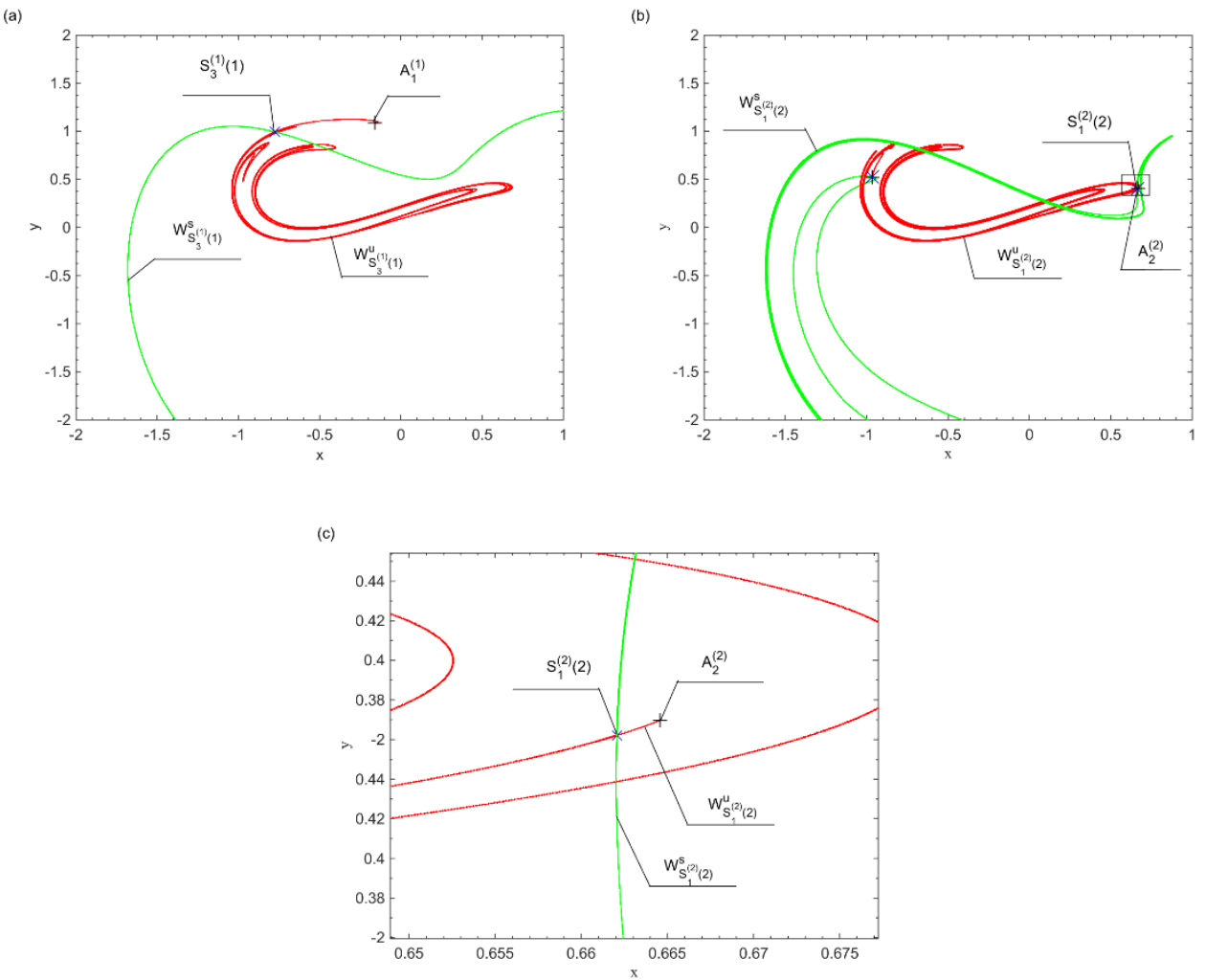

Fig. 8. Stable and unstable manifolds of saddles (a) $S_{3}^{(1)}(1)$, (b) $S_{1}^{(2)}$ (2). (c) is the local enlargement of the rectangle area of (b).

\subsection{CSC bifurcation}


Note that the evolution of two different chaotic attractors $A_{2}^{(\infty)}$ and $A_{3}^{(\infty)}$ implies the occurrence of complex bifurcation associated with chaotic saddle. In this subsection, we focus on the analysis of CSC bifurcation as $\omega \in\left[\omega_{\mathrm{SN}}, \omega_{\mathrm{PD}}\right]$.

It is obvious from Fig. 6 that no bifurcation occurs when $\omega$ increases from $\omega_{\mathrm{SN}}$ to $\omega_{\mathrm{PD}}$. However, CSC bifurcation takes place when $\omega$ is close to 1.1389. Fig. 9 shows the global properties. The clear fractal structure appears in Fig. 9. When $\omega=1.1388<\omega_{\mathrm{CSC}}$, right before CSC bifurcation, there is an unique chaotic saddle $\mathrm{S}_{2}^{(\infty)}(1,2)$ (red dots) located on the boundary of basin $\mathrm{B}(1)$ (blue dots) and $\mathrm{B}(2)$ (green dots), see Fig. 9(a). The basin boundary is denoted by $\mathrm{B}(1,2)$ (white area in Fig. 9). For $\omega=1.1389 \approx \omega_{\mathrm{CSC}}$, right after CSC bifurcation, $\mathrm{BCS} \mathrm{S}_{2}^{(\infty)}(1,2)$ undergoes a rupture and is decomposed into two distinct chaotic saddles denoted by BCS $\mathrm{S}_{4}^{(\infty)}(1,2)$ (red dots) and two-branch ICS $\mathrm{S}_{5}^{(\infty)}(2)$ (blue dots), see Fig. 9(b).
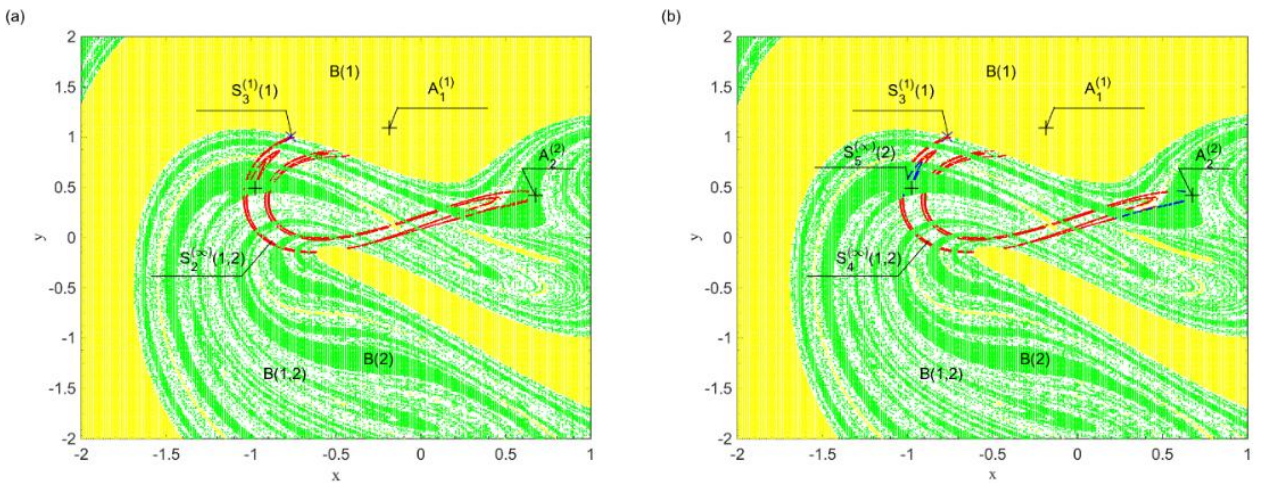

Fig. 9. Global properties for (a) $\omega=1.1388<\omega_{\mathrm{CSC}}$, (b) $\omega=1.1389 \approx \omega_{\mathrm{CSC}}$.

In order to unfold the mechanism of this CSC bifurcation, stable and unstable manifolds of period saddles are exhibited computationally. When $\omega$ is close to $\omega_{\mathrm{CSC}}$, the period saddle $S_{6}^{(4)}(1,2)$, which is embedded on the boundary of chaotic saddles, plays a significant role for the above CSC bifurcation.

For $\omega=1.1388<\omega_{\mathrm{CSC}}$, Fig. 10(a) and (b) show two different directions (left and right branches) of stable manifold $W_{\mathrm{S}_{6}^{(4)}(1,2)}^{S}$ (red dots) and unstable manifold $W_{\mathrm{S}_{6}^{(4)}(1,2)}^{u}$ (blue dots) of period saddle $\mathrm{S}_{6}^{(4)}(1,2)$. These two unstable manifolds 
mediate eventually at attractor $\mathrm{A}_{2}^{(2)}$. It can be seen clearly from Fig. 10(a), the left branch of unstable manifold resembles $\operatorname{BCS~}_{2}^{(\infty)}(1,2)$ in Fig. 9(a). Moreover, it intersects transversally the left branch of stable manifold. This intersection implies wrap structure and can eventually induces the generation of $\operatorname{BCS} S_{2}^{(\infty)}(1,2)$. Note that, $\operatorname{BCS} S_{2}^{(\infty)}(1,2)$ in Fig. 9(a) have some gaps. The space between intersection points in Fig. 10(a) is the origin of these gaps. These gaps reflect the fractal structure in Fig. 9, this phenomenon is similar with the results in [21]. Fig. 10(b) shows the right branch of unstable manifold. This unstable manifold also intersects with another stable manifold. The local enlargement of Fig. 10(b) is present in Fig. 10(e). The abbreviated symbols $W_{i}^{s}$ and $W_{i}^{u}(i=1,2,3,4)$ represent stable and unstable manifolds for the $i$ th point of saddle $S_{6}^{(4)}(1,2)$. Fig. 10(e) exhibits the intersection between unstable manifold $W_{4}^{u}$ and stable manifold $W_{1}^{s}$. This intersection reveals that the trajectory on the right branch of unstable manifold $W_{4}^{u}$ can cross stable manifold $W_{1}^{S}$, going along the left branch of unstable manifold and back to the right branch of unstable manifold. Wrap caused by intersections induces the combination between the left and right branches of unstable manifold in Fig. 10(a) and (b).

For $\omega=1.1389 \approx \omega_{\mathrm{CSC}}$, right after CSC bifurcation, Fig. $10(\mathrm{c})$ and (d) also exhibit the left and right branches of manifolds. Their outlines resemble Fig. 10(a) and (b), respectively. Two branches of unstable manifolds also mediate eventually at attractor $A_{2}^{(2)}$. Fig. 10(f) is the local enlargement of Fig. 10(d). Compare with Fig. 10(e), Fig. 10(f) shows no intersection between stable manifold and unstable manifold. This divided structure interrupts the access of orbits from the unstable manifold to the stable manifold. It reveals that the left and right branches of unstable manifolds in Fig. 10(c) and (d) are disconnected. This disconnection induces the creation of two invariant sets named BCS $S_{4}^{(\infty)}(1,2)$ and $\operatorname{ICS~} S_{5}^{(\infty)}(2)$. At CSC bifurcation saddle $\mathrm{S}_{6}^{(4)}(1,2)$ fill the gaps between these two chaotic saddles. 
(a)

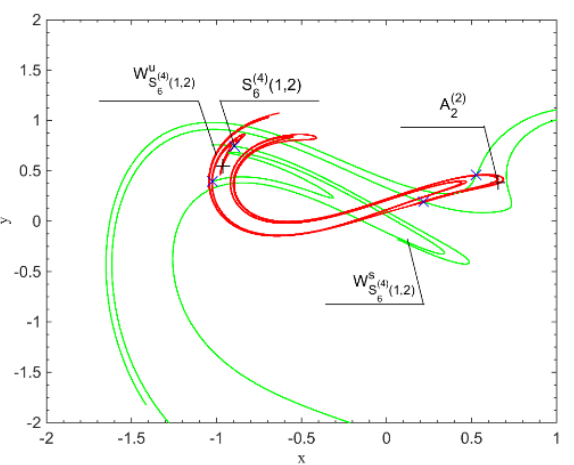

(c)

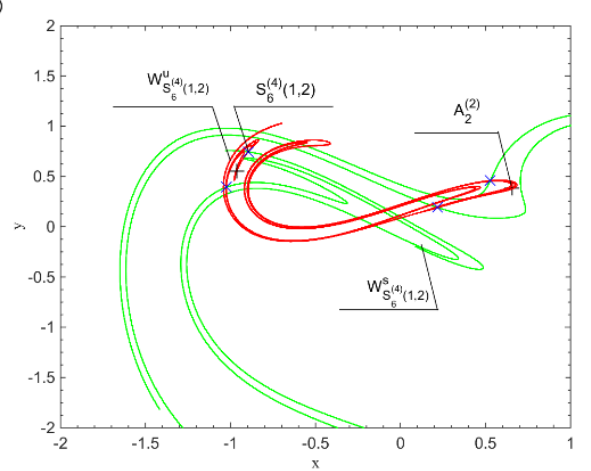

(e)

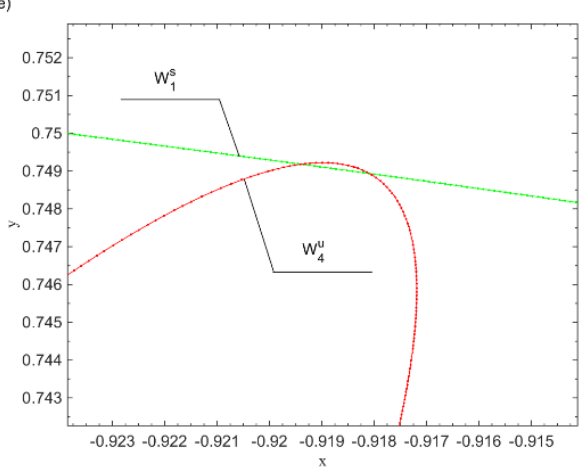

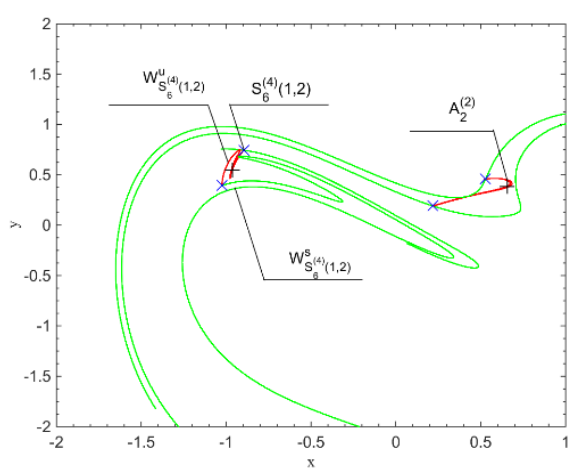
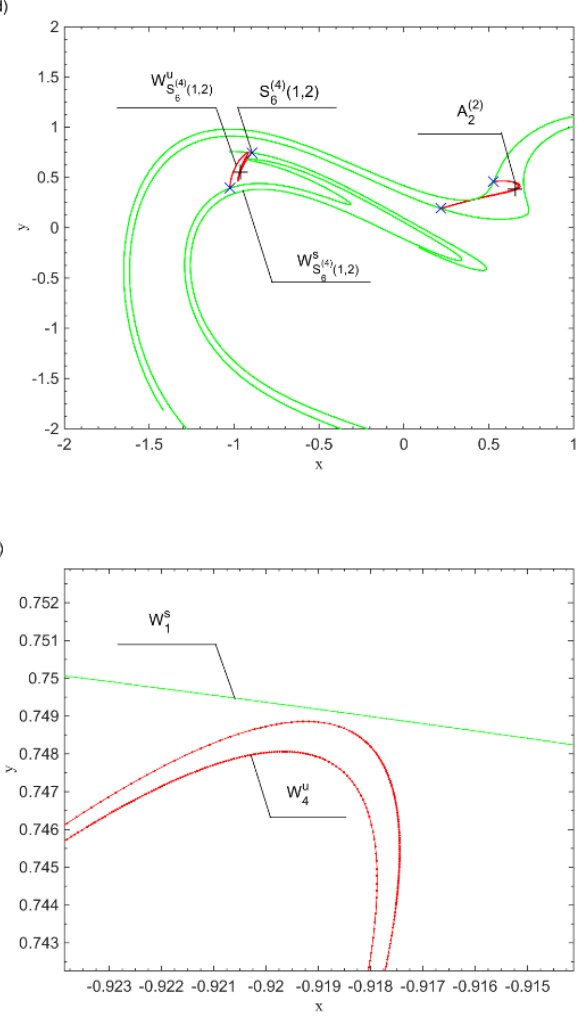

Fig. 10. Stable manifold $W_{\mathrm{S}_{6}^{(4)}(1,2)}^{S}$ and unstable manifolds $W_{\mathrm{S}_{6}^{(4)}(1,2)}^{u}$ of period saddles $\mathrm{S}_{6}^{(4)}(1,2)$. (a) left branch manifold for $\omega=1.1388<\omega_{\mathrm{CSC}}$. (b) right branch manifold for $\omega=1.1388<$ $\omega_{\mathrm{CSC}}$. (c) left branch manifold for $\omega=1.1389 \approx \omega_{\mathrm{CSC}}$. (d) right branch manifold for $\omega=$ $1.1389 \approx \omega_{\mathrm{CSC}}$. (e) and (f) are the local enlargement of (b) and (d), respectively.

\subsection{ICC bifurcation}

In this subsection, we focus our interest on the interior chaotic crisis bifurcation. Just before ICC bifurcation $\omega=1.1423<\omega_{\text {ICC }} \approx 1.1424$, there are no chaotic attractors. Fig. 11(a) show $\operatorname{BCC~} S_{4}^{(\infty)}(1,2)$ and $\operatorname{ICS~} S_{5}^{(\infty)}(2)$ denoted by red dots 
and blue dots, respectively. Compare with Fig. 9(b), the factual structure of these two chaotic saddles become more pronounced due to the increase of size of its gaps. For $\omega=1.1424 \approx \omega_{\text {ICC }}$, right after ICC bifurcation, two-band ICS $S_{5}^{(\infty)}(2)$ changes into chaotic attractor $A_{3}^{\infty}$ denoted by black dots, see Fig. 11(b).
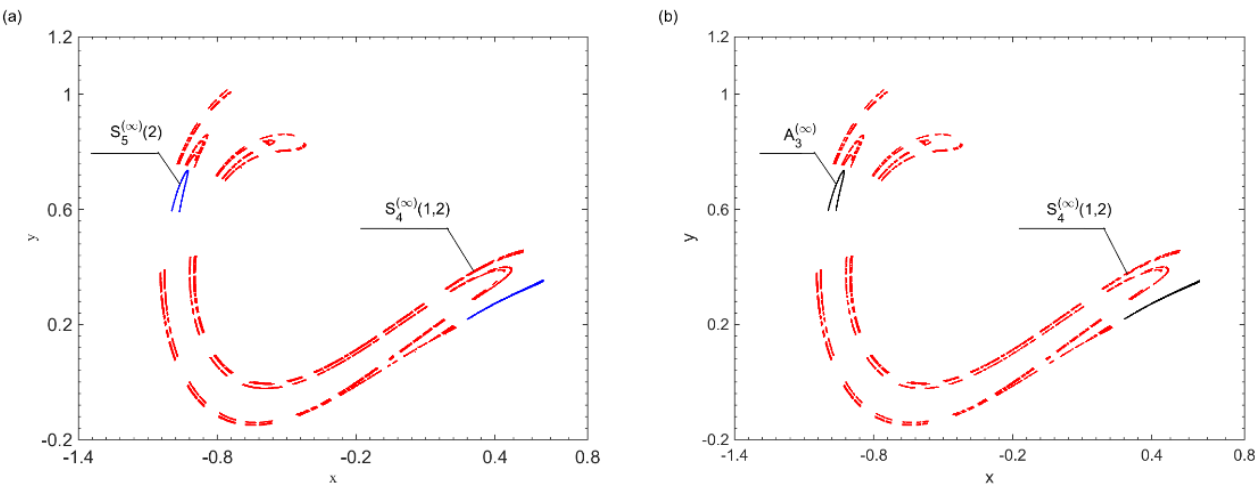

Fig. 11. Chaotic saddles and chaotic attractor before and after ICC bifurcation. (a) $\omega=1.1423<$ $\omega_{\mathrm{ICC}}$, (b) $\omega=1.1424 \approx \omega_{\mathrm{ICC}}$.

Previous results show that this ICC bifurcation is usually caused by the collision between chaotic attractor and unstable orbits, see Ref. [22]. In fact, the intersection between stable and unstable manifolds of period saddle is responsible for this transition. Before and after ICC bifurcation, stable and unstable manifolds (denoted by green and red dots) are shown in Fig. 12(a) and (b), respectively. The complete stable manifolds of period saddle $S_{1}^{(2)}$ fill the gaps of BCS $S_{4}^{(\infty)}(1,2)$. The local enlargements Fig. 12(c)-(f) exhibit clearly that the structure of stable and unstable manifolds changes from the intersection to the disconnection as the increase of $\omega$ from 1.1323 to 1.1424 . Before ICC bifurcation, the intersection between stable manifold and one branch of unstable manifolds can create an invariant set, see Fig. 12(c) and (e). Through these intersection points, the orbits of this invariant set can gain access to stable manifold, and eventually escape from saddle $\mathrm{S}_{1}^{(2)}$ to attractor $\mathrm{A}_{2}^{(2)}$ along another direction of unstable manifold. This nonattracting invariant set consists of BCS $\mathrm{S}_{4}^{(\infty)}(1,2)$. 
As the increase of $\omega$ to 1.1424, right after ICC bifurcation, Fig. 12(d) and (f) show no intersection between stable and unstable manifolds. This disconnection cut the access from unstable manifold to stable manifold. Chaotic orbits belonging to attractor are confined in the region occupied by the unstable manifold. These chaotic orbits can form chaotic attractor $A_{3}^{\infty}$, which is the closure of one branch of unstable manifold of saddle $S_{1}^{(2)}$.
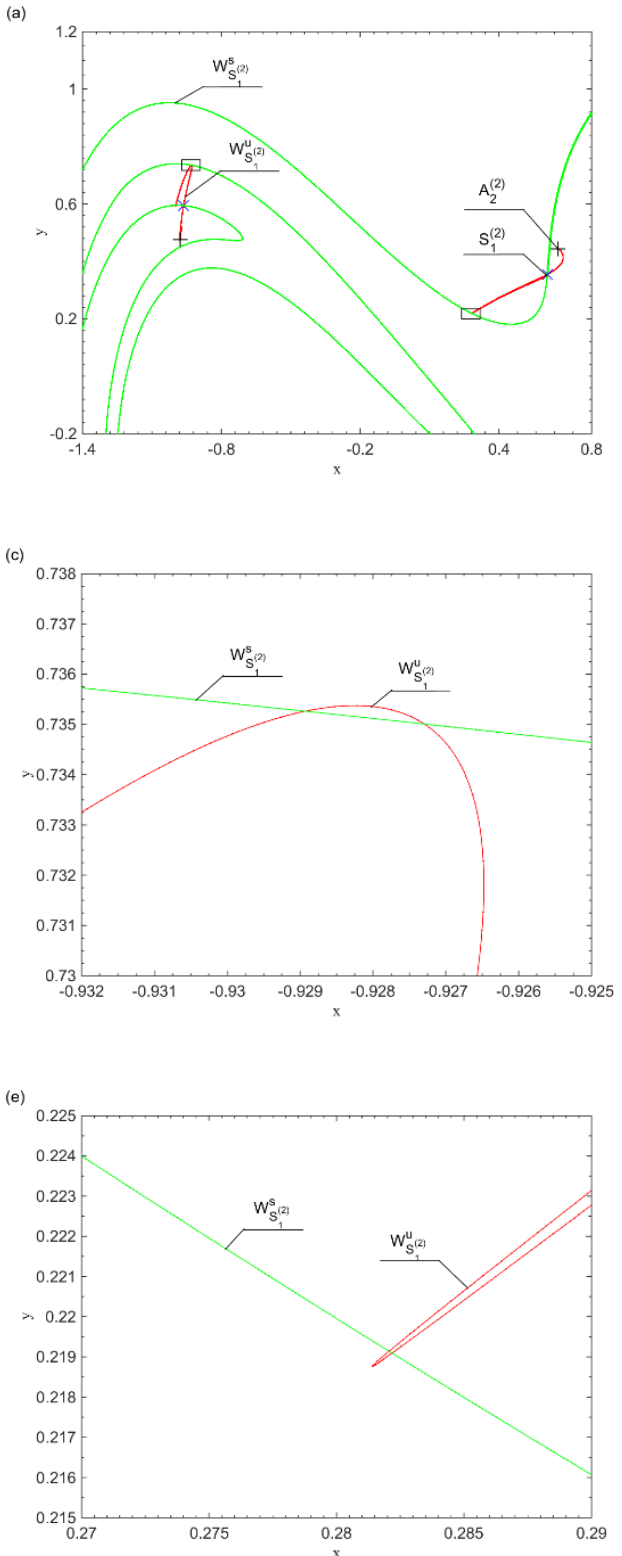

(b)

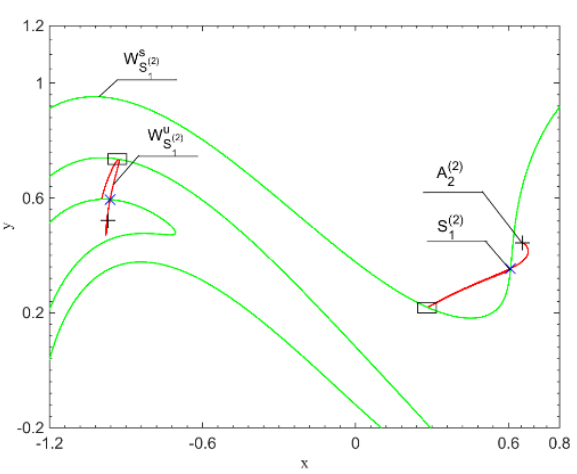

(d)

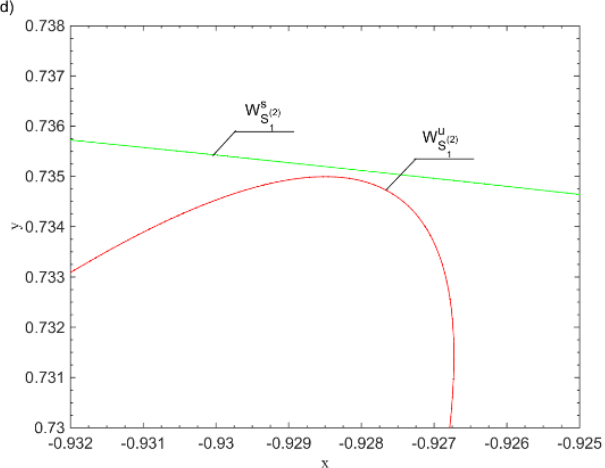

(f)

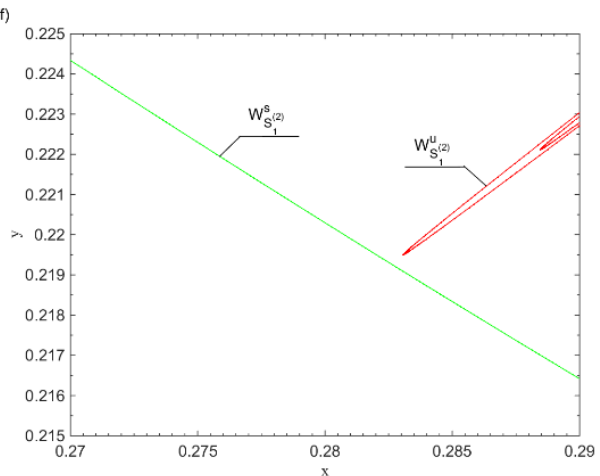

Fig. 12. Stable manifold $W_{\mathrm{S}_{1}^{(2)}}^{s}$ and unstable manifolds $W_{\mathrm{S}_{1}^{(2)}}^{u}$ of period saddles $\mathrm{S}_{1}^{(2)}$. (a) $\omega=1.1423<\omega_{\mathrm{IN}}$. (b) $\omega=1.1424 \approx \omega_{\mathrm{IN}}$. (c) and (e) are enlargements of the left and right rectangles of (a), respectively. (d) and (f) are enlargements of the left and right rectangles of (b), 
respectively.

\section{Conclusions}

It is well known that the chaotic saddle play a key role in dynamics of the chaotic transient, the chaotic crisis, the fractal basin boundary and the scattering. Using the improved stagger-and-step method, we are able to generate arbitrary long trajectory lying in a $\varepsilon$ neighborhood of the chaotic saddle. The paper is also interested in the analysis of chaotic saddles. Three types of bifurcation, including SN bifurcation, CSC bifurcation and ICC bifurcation, are investigated in detail.

At SN bifurcation, one branch of unstable manifold of the created saddle intersect its stable manifold and forms a chaotic saddle. Another unstable manifold can add a new escape route for this chaotic saddle. Two different escape routes cause the switch for this chaotic saddle to the boundary structure. At CSC bifurcation, the saddle at the link between two chaotic saddles plays important role. The disconnection of one branch of unstable and stable manifolds of this saddle can break the combination of two chaotic saddle. It eventually induces that the large chaotic saddle can divide into two different chaotic saddle. At ICC bifurcation, no intersection between unstable and stable manifolds can confine the chaotic orbits in the region occupied by this unstable manifold. This chaotic orbits then form a closed set and change into a chaotic attractor from a chaotic saddle with escape route.

The intersection between stable and unstable manifolds of saddles is responsible for the formation of gaps in the chaotic saddle. This gaps is important to explain the mechanism of fractal structure.

\section{Acknowledgements}

The authors are grateful to the support of National Science Foundation of China (Grant No. 11302158) and Natural Science Foundation of Shannxi Province, China (Grant No. 2015JM1034). 


\section{Reference}

[1] Shaw SW, Holmes PJ. Periodically forced linear oscillator with impacts-chaos and long period motions. Phys. Rev. Lett. 1983; 51: 623-626.

[2] Rong HW, Wang XD, Xu W, and Fang T, Rhys. Rev. E 2009; 80: 0266041-0266048.

[3] di Bernardo M, Garofalo F, Glielmo L, Vasca L. Swithchings, bifurcations and chaos in DC/DC converters. IEEE Transactions on Circuits and Systems, Part I 1998; 45:133-141.

[4] van der Schaft AJ, Schumacher JM. An introduction to Hybrid Dynamical Systems. Springer-Verlag, New York, 2000.

[5] Kunze M. Non-Smooth Dynamical Systems, Springer, Berlin, 2000.

[6] Leine RI, Nijmeijer H. Dynamics and Bifurcations in Non-Smooth Mechanical Systems. Springer-Verlag, Berlin, 2004.

[7] di Bernardo M, Budd CJ, Champneys AR, Kowalczyk P. Piecewise-smooth Dynamical Systems: Theory and Applications, Springer-Verlag, 2007.

[8] Luo ACJ, Gegg BC. Grazing phenomena in a periodically forced, friction-induced, linear oscillator. Commun Nonlinear Sci Numer Simulat 2006; 11: 777 - 802.

[9] Piiroinen PT, Virgin LN, Champneys AR. Chaos and period-adding; experimental and numerical verification of the grazing bifurcation. Journal of Nonlinear Science 2004; 14(4): 627-654.

[10] di Bernardo M, Budd CJ, Champneys AR. Grazing and border-collision in piecewise smooth systems: a unified analytical framework, Phys. Rev. Lett. 2001; 86: 2553-2556.

[11] Awrejcewicz J, Sendkowski D. How to predict stick-slip chaos in $R^{4}$. Physics Letters A 2004;330: 371-376.

[12] Mason JF, Piiroinen PT, Wilson RE, Homer ME. Basins of attraction in nonsmooth models of gear rattle. International Journal of Bifurcation and Chaos 2009; 19 (01): 203-224.

[13] Mason JF, Piiroinen PT. Interactions between global and grazing bifurcations in an impacting System. Chaos 2011; 21: 013113-9.

[14] Ott E. Chaos in Dynamical Systems (second edition), Cambridge University, Cambridge, 2002.

[15] Yue XL, Xu W, Wang L. Global analysis of boundary and interior crises in an elastic impact oscillator. Commun Nonlinear Sci Numer Simulat 2013; 18 (12): 3567-3574.

[16] Feng JQ, Xu W, Rong HW, Wang R. Stochastic responses of Duffing-Van der Pol vibro-impact system under additive and multiplicative random excitations. International Journal of Non-Linear Mechanics 2009; 44: 51-57.

[17] Hsu GH, Ott E, Grebogi C. Strange saddles and the dimensions of their invariant manifolds. Phys. Lett. A 
1988; 127 : 199-204.

[18] Nusse HE, Yorke JA. A procedure for finding numerical trajectories on chaotic saddles, Physica D 1989;36: $137-156$.

[19] Moresco P, Dawson SP. The PIM-simplex method: an extension of the PIM-triple method to saddles with an arbitrary number of expanding directions. Physica D 1999; 126: 38-48.

[20] Sweet D, Nusse HE, Yorke JA. Stagger-and-step method: detecting and computing chaotic saddles in higher dimensions. Phys. Rev. Lett. 2001; 86: 2261-2264.

[21] Rempel EL,Chian AC-L, Macau EEN, et al.. Analysis of chaotic saddles in high-dimensional dynamical systems:The Kuramoto-Sivashinsky equation. Chaos 2004; 14(3): 545-556.

[22] Tel T, Lai YC. Chaotic transients in spatially extended systems. Physics Reports 2008; 460: 245-275. 